\section{Intersections}

Canadian Journal of Music

Revue canadienne de musique
Intersections CANADIAN JOURAL OF MUSIC
REVUE CANADIENEE DE MUSIOUH

\title{
Cadre pédagogique pour l'enseignement-apprentissage de l'improvisation musicale classique fondé sur la pratique des experts du domaine
}

\author{
Jean-Philippe Després, Pamela Burnard, Francis Dubé et Sophie Stévance
}

Volume 35, numéro 2, 2015

URI : https://id.erudit.org/iderudit/1043820ar

DOI : https://doi.org/10.7202/1043820ar

Aller au sommaire du numéro

Éditeur(s)

Canadian University Music Society / Société de musique des universités canadiennes

ISSN

1911-0146 (imprimé)

1918-512X (numérique)

Découvrir la revue

Citer cet article

Després, J.-P., Burnard, P., Dubé, F. \& Stévance, S. (2015).

Cadre pédagogique pour l'enseignement-apprentissage de l'improvisation musicale classique fondé sur la pratique des experts du domaine. Intersections, 35(2), 3-36. https://doi.org/10.7202/1043820ar
Résumé de l'article

Cette recherche porte sur l'enseignement-apprentissage de l'improvisation musicale classique. Afin d'offrir une perspective en profondeur de cet objet d'étude, des entrevues semi-dirigées ont été menées auprès de $\mathrm{N}=15$ participants, au sujet de leur processus d'apprentissage, de production et d'enseignement de l'improvisation. Différentes composantes de l'enseignement-apprentissage de l'improvisation musicale classique ont été identifiées : 18 compétences à développer chez l'apprenant et 23 approches d'enseignement-apprentissage. Ces connaissances offrent à celui qui s'intéresse à l'improvisation - qu'il soit enseignant ou apprenant, de niveau novice, débutant ou intermédiaire - des balises pour guider son apprentissage instrumental ou sa pratique pédagogique.
Copyright (C Canadian University Music Society / Société de musique des universités canadiennes, 2018
Ce document est protégé par la loi sur le droit d'auteur. L'utilisation des services d’Érudit (y compris la reproduction) est assujettie à sa politique d'utilisation que vous pouvez consulter en ligne. 


\title{
CADRE PÉDAGOGIQUE POUR L'ENSEIGNEMENT- APPRENTISSAGE DE L'IMPROVISATION MUSICALE CLASSIQUE FONDÉ SUR LA PRATIQUE DES EXPERTS DU DOMAINE
}

\author{
Jean-Philippe Després, Pamela Burnard, Francis Dubé, et Sophie \\ Stévance
}

\section{INTRODUCTION}

\begin{abstract}
Improvisation and composition are two different art forms. Improvisation is "composition become performance». The danger: one plays what one knows. Don't play safe. Play what you don't know. Avoid Formulae - take a chance. (Lukas Foss, cité par Campbell 1991, p. 23)
\end{abstract}

Si l'improvisation n'est actuellement pratiquée que par une poignée de musiciens classiques, il n'en a pas toujours été ainsi. En effet, l'improvisation était une habileté indispensable pour la plupart des musiciens de tradition classique jusqu'à la fin du XIX siècle (Moore 1992; Scott 2007). Toutefois, depuis environ 200 ans, l'improvisation a progressivement disparu de la pédagogie et de la scène musicale classique (Moore 1992). L’une des causes attribuées à ce phénomène est liée aux exigences techniques sans cesse croissantes du répertoire joué par les musiciens. En effet, afin de développer la virtuosité requise pour interpréter les œuvres de concert, les instrumentistes des XIX ${ }^{\mathrm{e}}$ et $\mathrm{XX}^{\mathrm{e}}$ siècles auraient délaissé peu à peu les autres compétences musicales, telles que l'improvisation et la composition (Sloboda 1993). Conséquemment, cette surspécialisation des musiciens classiques aurait entraîné une importante diminution de leur polyvalence, en accentuant le clivage entre les rôles de compositeur, d'interprète et d'improvisateur (Berkowitz 2009; Manderson 2010; Moore 1992).

En revanche, depuis quelques décennies, les efforts conjugués de chercheurs, de musicologues et d'interprètes ont ravivé l'intérêt pour l'improvisation musicale classique (Solis et Nettl 2009). Cette résurgence est porteuse d'un immense potentiel pédagogique, puisque l'apprentissage de l'improvisation exerce un effet positif sur l'acquisition et le développement de plusieurs autres compétences musicales (Azzara 1992; Dos Santos et Del Ben 2004; Kenny et Gellrich 2002; Koutsoupidou et Hargreaves 2009; McPherson 1993; Montano 1983; Whitman 
2001; Wilson 1970) (voir Dubé et Després 2012 pour une analyse détaillée de cette littérature). Ainsi, l'intégration de l'improvisation au curriculum classique permettrait de former des musiciens plus polyvalents et mieux outillés pour répondre aux exigences de leur future vie professionnelle. En revanche, l'état actuel des connaissances dans le domaine de l'improvisation musicale ne permet pas de pourvoir adéquatement le pédagogue ou les institutions souhaitant intégrer l'improvisation au curriculum musical classique (Després 2011). En effet, en dépit du vibrant plaidoyer pour la réintroduction de l'improvisation dans la pédagogie musicale classique, cette dernière est toujours très peu enseignée dans les établissements d'enseignement postsecondaire de la musique (McPherson et al. 1997).

Différents facteurs contribuent à ce clivage entre la situation actuelle dans les établissements d'enseignement et le scénario préconisé par les chercheurs. Tout d'abord, la plupart des enseignants en musique classique ont reçu une formation traditionnelle, de type conservatoire, où - sauf chez les organistes ${ }^{1}-$ l'improvisation n'est pas enseignée (Sloboda 1996). Comme les enseignants ont tendance à adopter des pratiques pédagogiques reflétant la façon dont ils ont eux-mêmes appris (Dubé, Héroux et Robidas 2015; Dunn et Dunn 1979), ils tendent simplement à ne pas enseigner l'improvisation à leurs élèves. En outre, un manque de ressources pédagogiques basées sur la pratique réelle des experts du domaine pourrait également contribuer à l'absence relative d'improvisation dans les établissements offrant une formation de niveau postsecondaire en musique classique (Dubé et Després 2012). Cette lacune pourrait être expliquée par le fait que les stratégies d'enseignement-apprentissage des experts dans le domaine de l'improvisation musicale classique n'ont pas, à ce jour, été documentées scientifiquement de manière exhaustive. Ainsi, pour contribuer à étayer les connaissances dans ce domaine, il s'avère nécessaire d'interroger des pédagogues et instrumentistes experts du domaine concernant leur approche d'apprentissage et d'enseignement de l'improvisation musicale. Ces connaissances, fondées sur l'expérience pratique des porteurs du savoir dans ce domaine, revêtiraient un grand potentiel pédagogique. En effet, une pédagogie qui repose sur une compréhension éclairée du mode de pensées des experts permettrait d'accélérer le processus d'apprentissage des apprenants, comme le remarque Norgaard (2011) dans son étude portant sur les processus mentaux d'experts en improvisation jazz:

L'objectif principal de l'enseignement de l'improvisation devrait être de structurer des activités d'apprentissage lors desquelles des élèves de tous les niveaux peuvent expérimenter des façons de penser qui ressemblent à celles des improvisateurs experts. Atteindre cet objectif exige que l'on examine de plus près et que l'on décrive plus clairement non seulement ce que les experts font, mais aussi la façon dont ils pensent à ce qu'ils font ${ }^{2}$. (p. 124)

1 En effet, l'improvisation est pratiquée de façon ininterrompue dans la tradition organistique depuis l'époque baroque («Improvisation» s.d.).

2 Traduction libre de : «The primary goal of improvisation instruction should be to structure learning activities in which students at all levels can experience ways of thinking that resemble those 
En résumé, la pédagogie de l'improvisation musicale classique pourrait être bonifiée par une meilleure compréhension des stratégies d'enseignement et d'apprentissage des experts du domaine. Cet éclairage permettrait de poser les fondations requises pour outiller l'enseignant qui souhaiterait intégrer efficacement l'improvisation à sa pratique pédagogique.

\section{ÉTAT DES CONNAISSANCES}

Plusieurs recherches empiriques ont démontré l'effet positif de l'apprentissage de l'improvisation musicale sur d'autres variables, telles que l'assimilation des concepts théoriques, la lecture musicale ou la qualité de la prestation (Azzara 1992 ; McPherson 1993), l'acuité rythmique (Montano 1983), les perceptions auditives (Dos Santos et Del Ben 2004; Whitman 2001; Wilson 1970), la créativité musicale (Koutsoupidou et Hargreaves 2009), l'aisance scénique, l'expressivité et la motivation (Kenny et Gellrich 2002; Sloboda 1993), ainsi que la relaxation et le timbre (Moreira et Carvalho 2010) et les sentiments d'authenticité et de présence (Lange 2011).

Quoique les effets positifs de l'apprentissage et la pratique de l'improvisation musicale aient été largement documentés, les pratiques d'enseignement et les modalités d'apprentissage de l'improvisation musicale classique demeurent largement méconnues. Afin de bien cerner le manque à combler et de situer notre recherche dans le contexte de l'état actuel des connaissances, la présente recension sera orientée autour des littératures théoriques et empiriques centrées sur l'enseignement-apprentissage de l'improvisation musicale.

\section{LITTÉRATURE THÉORIQUE}

Le modèle de Kratus $(1991,1995)$ propose une séquence de sept étapes d’apprentissage de l'improvisation. Les sept phases de son modèle sont les suivantes:

1. exploration: phase préparatoire à l'apprentissage de l'improvisation où l'apprenant expérimente des combinaisons sonores dans un contexte peu structuré;

2. improvisation axée sur le processus: l'apprenant commence à répéter des motifs cohérents;

3. improvisation axée sur le produit: l'apprenant fait appel à des principes structurels d'organisation tels que la tonalité, la pulsation et la métrique;

4. improvisation fluide: l'apprenant a automatisé suffisamment d'habiletés techniques pour improviser de manière plus aisée;

5. improvisation structurelle: l'apprenant possède un répertoire de stratégies lui permettant de donner une structure formelle à son improvisation; 
6. improvisation stylistique: l'apprenant improvise en respectant les caractéristiques harmoniques, mélodiques et rythmiques d'un style donné;

7. improvisation personnelle: phase rarement atteinte où le musicien transcende les styles connus pour développer un style personnel, avec son propre système de règles et de conventions. (Traduction et explications tirées de Dubé et Després 2012, p. 153-154)

En somme, le modèle de Kratus permet de faciliter l'évaluation du niveau de l'apprenant ainsi que la planification des contenus à enseigner en conséquence.

Kenny et Gellrich (2002) ont développé un modèle théorique de l'apprentissage de l'improvisation qui comprend quatre phases: (1) l'apprentissage des hardware, (2) l'apprentissage des software, (3) la stimulation de la créativité et (4) l'improvisation associative, en plus de souligner l'importance de jouer et d'analyser des compositions musicales pour apprendre à improviser (Kenny et Gellrich 2002, p. 132). Ce modèle illustre la complexité du processus d'apprentissage de l'improvisation musicale et le fait qu'une approche pédagogique de l'improvisation devrait adopter une perspective plurielle et holistique.

Thompson et Lehmann (2004) proposent diverses stratégies pouvant favoriser l'acquisition d'habiletés en improvisation. Par exemple, ils suggèrent que le musicien devrait:

- Bien connaître le style dans lequel il cherche à improviser; écouter de la musique, analyser des partitions et étudier des prestations d'interprètes professionnels pour se familiariser à ce style;

- S'exercer à prendre conscience de son activité mentale alors qu'il improvise;

- Créer ses propres exercices techniques afin d'apprendre à extraire du répertoire les aspects essentiels à travailler;

- Chanter en improvisant afin de favoriser la création de liens entre ses habiletés perceptuelles, cognitives et motrices;

- Ne pas se décourager et prendre conscience que l'improvisation demande beaucoup d'efforts cognitifs au début, mais qu'elle finit par s'automatiser;

- S'imposer des contraintes artificielles afin de briser ses habitudes, surtout si son improvisation manque d'originalité;

- Apprendre à prendre des risques.

Bien que l'effet des stratégies proposées par Thompson et Lehmann (2004) n'ait pas été validé scientifiquement, leurs propositions, en plus d'offrir des pistes pour l'apprenant ou l'enseignant qui souhaiterait intégrer l'improvisation à sa pratique, laissent entrevoir, à l'instar du modèle de Kenny et Gellrich (2002), le fait qu'une pédagogie de l'improvisation devrait être multiple et intégrative.

En résumé, la recherche théorique sur l'improvisation musicale offre des pistes de réflexion pour l'enseignant qui souhaiterait intégrer l'improvisation à sa pratique pédagogique, mais elle est fondée sur l'intuition des auteurs ou leur analyse de la littérature scientifique sur le sujet. Ainsi, elle présente les lacunes de ne pas être informée par le vécu des acteurs clés du domaine et de ne pas 
reposer sur des données probantes, lesquelles sont nécessaires pour mesurer l'effet réel des propositions pédagogiques proposées dans ce corpus sur l'apprenant. De plus, les considérations stylistiques et les éventuelles différences entre les genres musicaux sont plus ou moins exclues du discours de ces écrits théoriques, puisqu'ils abordent le plus souvent l'improvisation de façon plutôt générale que spécifique.

\section{LITTÉRATURE EMPIRIQUE}

Des recherches empiriques menées dans divers contextes (jazz, improvisation libre, improvisation vocale, et musique classique) et auprès de populations expertes, instrumentistes ou enseignants, se sont intéressées de près ou de loin au processus d'enseignement-apprentissage de l'improvisation musicale.

\section{En jazz}

Fraser (1983), dans sa thèse doctorale, a identifié cinq étapes d'apprentissage chez les improvisateurs jazz: (1) un attrait pour la musique jazz; (2) la formation auditive et l'observation; (3) la musique en soi et l'apprentissage instrumental; (4) l'imitation de modèles et le raffinement; (5) l'actualisation de soi et le développement stylistique individuel.

Berliner (1994) a cherché à documenter l'éventail des activités musicales des experts en improvisation jazz. Pour ce faire, il a effectué une importante revue de littérature, mené des entretiens avec plus d'une cinquantaine de musiciens, assisté à plusieurs répétitions et concerts, recommencé à suivre des leçons de trompette jazz et réalisé des transcriptions et des analyses d'enregistrements jazz. Alors qu'il documente de façon exhaustive les multiples dimensions de son objet d'étude, il n'offre pas de synthèse de ses résultats qui permettrait d'avoir une vue d'ensemble du parcours d'apprentissage des musiciens jazz. Ses résultats de recherche montrent néanmoins que le processus d'apprentissage des improvisateurs jazz est complexe et médiatisé par différents facteurs, tels que : l'enculturation, la participation au sein de la communauté jazz, l'assimilation délibérée de modèles et du répertoire, le développement des habiletés auditives et instrumentales, l'acquisition de connaissances musicales et théoriques, une routine de répétition, ainsi que des expériences de concerts fréquentes.

\section{Improvisation libre}

L'improvisation libre recèle un grand potentiel pédagogique, notamment parce que tous les apprenants peuvent la pratiquer, quel que soit leur niveau (Hickey 2015). Johnston (2013) a interviewé les membres de l'ensemble Jimmy Giuffre $3^{3}$, afin de proposer des approches alternatives pour enseigner l'im-

3 Jimmy Giuffre led several different drummer-less trios under the name The Jimmy Giuffre 3 throughout his career, the best known of which featured guitarist Jim Hall and either trombonist Bob Brookmeyer or bassist Ralph Peña. This version of the Giuffre 3 had a jazz hit in the late 1950 s with a tune called 'The Train and the River'. My focus is on Giuffre's subsequent trio with pianist Paul Bley and bassist Steve Swallow, which was his primary musical project from 1960 to 1962. Giuffre, Bley, and Swallow - in direct response to the pioneering work of Ornette Coleman — participated in the avant- 
provisation au niveau postsecondaire. Sans toutefois proposer de modèle ou de séquence pédagogique clairement articulée, l'auteur explique que, même dans le cadre de l'improvisation "libre», des "prémisses» (plus communément appelées «contraintes») sont établies par les musiciens avant d'improviser afin d'encadrer leur improvisation. Les prémisses qu'il a identifiées sont les suivantes: (a) l'empreinte d'un style musical; (b) déroger des rôles traditionnels des instruments; (c) expérimenter différentes approches avec la pulsation (par exemple, avec pulsation, sans pulsation ou avec plusieurs pulsations différentes); (d) expérimenter différentes approches de voicing (par exemple, tous les instruments dans le même registre ou tous les instruments dans des registres les plus éloignés possible). Ces différentes «prémisses» étaient explorées en profondeur lors des répétitions de l'ensemble avec l'objectif d'élargir leur spectre des possibles en concert.

Hickey (2015) a étudié l'approche pédagogique de quatre experts qui enseignement l'improvisation libre au niveau universitaire afin d'en extraire des éléments applicables dans le contexte de l'éducation musicale. L'auteure s'intéresse à l'improvisation libre, car cette pratique offre, selon elle, l'avantage de permettre aux apprenants de tous les niveaux d'improviser et de faire l'expérience d'une pratique musicale créative, sans nécessiter le bagage qui est généralement requis pour pratiquer d'autres formes d'improvisation. Suite à l'analyse de ses données, Hickey (2015) conclut:

Les thèmes communs qui ont émergé chez les quatre pédagogues comprennent un éventail d'exercices pédagogiques uniques, une aisance avec le vocabulaire non traditionnel, la création d'un espace d'enseignement sécurisant et égalitaire, l'absence d'évaluation, le leader qui agit comme un guide, une facilité avec la spontanéité, et le pédagogue agissant comme interprète/improvisateur ${ }^{4}$. (p. 425)

Parmi les activités pédagogiques recensées par l'auteure, on retrouve: (a) jouer de longues notes (ou des silences) sur une pulsation fixe et (b) créer une boucle (loop) musicale et la modifier progressivement. Par ailleurs, différentes recommandations pédagogiques pour l'enseignant découlent de l'analyse de ses données: (a) utiliser un vocabulaire métaphorique, en faisant peu appel

garde jazz scene in New York City in the early 196os that included musicians such as Cecil Taylor, Don Ellis, Carla Bley, George Russell, and Bill Dixon, among others. These musicians were concerned with finding ways to structure improvised performances that avoided the compositional forms, harmonic progressions, rhythmic schemes, and melodic structures that typified jazz of the late 1950s. In their brief time together the Jimmy Giuffre 3 recorded three studio albums: Fusion (1961) and Thesis (1961) on Verve Records, and Free Fall (1962) on Columbia. The music on these albums is an important link between the song and pulse-based free jazz of Ornette Coleman and Albert Ayler, and the less-structured music of the European free improvisers who emerged in the late 196os, such as Derek Bailey, Evan Parker, Peter Brötzmann, and Eddie Prévost. Giuffre, Bley, and Swallow disbanded their trio in 1962, reuniting briefly in the early 1990 os before Giuffre became too ill to perform; my investigation will focus on the trio's initial period of activity. (Johnston 2013, p. 386)

4 Traduction libre de: «The common themes that emerged among the four pedagogues included an array of unique teaching exercises, facility with nontraditional vocabulary, the establishment of a safe and egalitarian teaching space, lack of evaluation, leader as guide, comfort with spontaneity, and pedagogue as performer/improviser». (p. 425) 
aux termes musicaux; (b) préconiser le travail en petits ensembles; (c) lors de la rétroaction et de l'évaluation, éviter de parler de la "qualité» de l'improvisation, afin d'adopter une approche constructive; le pédagogue devrait plutôt décrire objectivement ce qui s'est passé et ce qu'il a ressenti lors de la prestation improvisée; (d) adopter un rôle de facilitateur; (e) adopter une approche pédagogique spontanée, à l'image de l'improvisation; (f) créer une atmosphère sécurisante et confortable pour l'apprenant; et (g) demeurer un improvisateur actif, notamment pour être en mesure de modéliser au besoin.

\section{1) Improvisation vocale}

Ward-Steinman (2014) s'est intéressée aux parcours d'apprentissage et aux pratiques pédagogiques de chanteurs improvisateurs-pédagogues professionnels œuvrant dans divers contextes stylistiques. Son analyse des données l'a menée à proposer différentes stratégies pédagogiques ainsi qu'un modèle pédagogique tripartite. En ordre d'importance quant au nombre d'occurrences, les stratégies d'apprentissages qu'elle a recensées sont les suivantes: (a) écouter et repiquer de «bons» albums; (b) chanter et mémoriser la fondamentale, les notes des accords et leurs extensions, les gammes et les mélodies de plusieurs standards jazz; (c) évoluer dans un environnement sécurisant, en focalisant son attention sur la musique plutôt que sur soi-même; (d) rechercher un équilibre le contrôle et la liberté, en explorant une grande variété de possibilités musicales; (e) trouver une voix personnelle; (f) développer la passion et la motivation nécessaires pour nourrir de bonnes habitudes de travail. Au cœur du modèle proposé par Ward-Steinman (2014) se trouve la motivation, nourrie par la valeur qui est attribuée à la musique et la possibilité d'évoluer dans un environnement sécurisant. Autour de la motivation se construit l'immersion progressive dans un style musical, médiatisée par des répétitions, les leçons, ainsi que l'écoute et le repiquage. Finalement, le fruit de ce processus est l'acte improvisé, une compétence qui est constituée des divers éléments musicaux et techniques acquis lors de la phase d'immersion.

\section{Jazz versus classique}

Kingscott et Durrant (2010) ont mené une étude comparant l'improvisation à l'orgue et l'improvisation jazz. Un musicien de haut niveau issu de chacune de ces traditions a été interviewé. Les résultats de cette étude montrent que, en jazz, l'enseignement de l'improvisation passe par la mémorisation d'idées musicales (licks) et par l'étude d'improvisations produites par d'autres musiciens. À l'orgue, l'écoute active occupe également une part importante de l'apprentissage de l'improvisation, de même que l'appropriation des normes stylistiques propres à un langage musical donné. Finalement, les auteurs concluent en affirmant que la principale implication pédagogique de leur étude est de démontrer l'importance d'offrir aux apprenants l'occasion d'improviser régulièrement en concert devant public, et non pas, comme c'est le cas dans la plupart des institutions, seulement au moment de l'évaluation. 


\section{En musique classique}

Trois études ont été menées sur l'enseignement-apprentissage de l'improvisation dans le contexte de la musique classique. La recherche de Pignato (2013) porte sur l'approche d'enseignement des pédagogues experts en improvisation dans le contexte de l'éducation musicale, tandis que celles de Berkowitz (2009) et de Johansson (2008) traitent, entre autres choses, du processus d'apprentissage de l'improvisation musicale classique de musiciens qui sont aujourd'hui des experts dans leur domaine.

D’abord, Pignato (2013) a étudié la réalité d'une éducatrice musicale qui a fait de l'improvisation une partie centrale de sa pratique. Les résultats de cette étude sont centrés sur les difficultés rencontrées par l'enseignante en raison de la marginalité de sa pratique, l'improvisation étant souvent mal reçue dans le milieu scolaire, par les parents des élèves et par certains élèves. Pour cette raison, les stratégies d'enseignement-apprentissage mises en place par l'enseignante sont très peu couvertes dans cette étude.

Ensuite, Berkowitz (2009) et Johansson (2008) ont étudié le développement de l'expertise à l'improvisation, respectivement dans le contexte de l'improvisation au clavier de la période classique et à l'orgue. Berkowitz (2009) a réalisé une analyse des traités de la période classique qui lui a permis d'identifier certains procédés utilisés à cette époque par les musiciens improvisateurs. Tout d'abord, il souligne l'importance capitale de la banque d'idées lors de l'improvisation. En effet, l'apprentissage de l'improvisation à l'époque classique passe notamment par l'internalisation de motifs en mémoire à long terme, lesquels sont destinés à être ensuite utilisés par le musicien lors de ses prestations. Cependant, lors de l'improvisation, le musicien ne peut pas se contenter d'enchâ̂ner de façon mécanique des motifs prémémorisés, il se doit donc de développer des stratégies qui lui permettent d'adapter et de modifier les motifs entreposés dans sa banque d'idées, telles que:

1. La transposition, souvent faite dans toutes les tonalités;

2. La variation d'une idée musicale;

3. La recombinaison, qui consiste à allier les caractéristiques de différents motifs en temps réel afin de produire une nouvelle idée musicale. (Berkowitz 2009)

En plus d'avoir analysé les traités d'époque, Berkowitz (2009) a interviewé deux musiciens d'aujourd'hui spécialisés en interprétation de la période classique qui improvisent en concert: Robert Levin et Malcolm Bilson. Ces entretiens ont d'abord révélé que ni un ni l'autre de ces musiciens n'a utilisé les traités d'époques accessibles pour apprendre à improviser. Ensuite, quatre processus d'apprentissage ressortent du parcours de ces deux musiciens: (1) la construction de la base de connaissances, par l'incubation/internalisation/assimilation du répertoire; (2) la compétence à utiliser et à enchaîner les idées contenues dans la base de connaissances, lors de répétitions personnelles; (3) le développement de l'aptitude en improvisation par les prestations devant public, qui créent des situations imprévues et un contexte communicatif nécessaires à l'apprentissage et (4) l'apprentissage à travers l'enseignement, qui incite le musicien à rationaliser des processus intuitifs afin de les transmettre aux apprenants. 
Enfin, la thèse doctorale de Johannson (2008) porte sur l'improvisation à l'orgue. Sa recherche a permis de mettre au jour quatre façons d'apprendre à improviser: (1) la fascination instrumentale, qui agit comme un motivateur important pour amorcer leur apprentissage initial; (2) l'apprentissage formel avec un maître; (3) l'apprentissage par la prestation, qui commence généralement assez tôt chez les organistes et qui est perçu par ces derniers comme étant la vraie manière d'apprendre et (4) la communication avec un guide intérieur, qui permet au musicien de s'autoévaluer et de continuer à progresser, même lorsque son apprentissage formel est achevé. Dans un contexte pédagogique, ces quatre façons d'apprendre pourraient être mises en valeur par l'adoption de différents rôles par l'enseignant:

1. Le guide, qui encourage l'élève et cherche à développer son intérêt pour l'instrument;

2. Le modèle, qui donne des exemples et transmet de l'information à l'élève;

3. Le challengeur, qui incite l'élève à appliquer ses connaissances dans des situations de concert réelles;

4. Le libérateur, qui incite l'élève à quitter la relation maître-élève et à développer une pratique musicale autonome. (Johansson 2008, p. 187-188)

En résumé, la littérature existante met en relief une pluralité de modalités d'enseignement-apprentissage de l'improvisation complémentaires et en partie idiosyncrasiques, pouvant être synthétisées comme suit:

1. un intérêt initial pour un instrument ou un genre musical (Fraser 1983; Johansson 2008);

2. un processus d'enculturation, par l'écoute, active ou non, l'analyse et l'imitation de modèles (Berliner 1994; Fraser 1983; Johansson 2008; Kenny et Gellrich 2002; Kingscott et Durrant 2010; Thompson et Lehmann 2004; Ward-Steinman 2014);

3. l'apprentissage des règles musicales et de la technique instrumentale (Berkowitz 2009; Berliner 1994; Fraser 1983; Johansson 2008; Kenny et Gellrich 2002; Kingscott et Durrant 2010);

4. la capacité d'utiliser et d'adapter ses connaissances et ses habiletés musicales en temps réel (Berkowitz 2009; Berliner 1994; Kenny et Gellrich 2002);

5. la mise en place de contraintes ou de règles d'improvisation (Hickey 2015; Johnston 2013; Kenny et Gellrich 2002; Thompson et Lehmann 2004);

6. un volet psychologique, comprenant des dimensions telles que la motivation, la prise de risques, l'auto-évaluation et l'autorégulation) (Berliner 1994; Johansson 2008; Thompson et Lehmann 2004);

7. une dimension sociale (Berliner 1994);

8. des apprentissages cristallisés dans un contexte de concert devant public ou d'enseignement (Berkowitz 2009; Berliner 1994; Johansson 2008; Kenny et Gellrich 2002); 
9. le développement d'un style personnel (Berliner 1994; Fraser 1983;

Kratus 1995; Ward-Steinman 2014).

Notre revue de la littérature a révélé un manque à combler sur le plan des connaissances liées aux approches d'enseignement-apprentissage et les stratégies de production des experts du domaine en musique classique. Ce manque a notamment comme conséquence de faire en sorte que les pratiques pédagogiques actuelles ne sont pas informées par les savoir-faire des principaux porteurs de la connaissance dans ces domaines (Després et Dubé 2015). Ainsi, afin de participer à étayer l'état des connaissances sur l'enseignement-apprentissage de l'improvisation musicale classique, nous formulons la question de recherche suivante: "Quels éléments liés à l'expérience, aux représentations, au parcours d'apprentissage ou à la pratique pédagogique des experts du domaine pourraient contribuer à bonifier l'enseignement-apprentissage de l'improvisation musicale classique?»

\section{OBJECTIFS DE RECHERCHE ET DÉFINITIONS OPÉRATIONNELLES}

Afin de répondre à notre question de recherche, nous avons formulé les objectifs suivants:

1. recenser les recommandations pédagogiques et les stratégies d'enseignement-apprentissage de musiciens et de pédagogues experts en improvisation musicale classique;

2. structurer les éléments recensés afin de formuler une proposition pédagogique cohérente.

Notre question et nos objectifs de recherche sont alignés sur notre intention de contribuer à la fois à l'avancement des connaissances dans le domaine du développement de l'expertise, dans le domaine de l'improvisation musicale classique et à l'établissement de ponts entre chercheurs et praticiens, par la diffusion de recommandations pédagogiques issues de nos résultats dans le milieu pratique. Conséquemment à notre question et à nos objectifs de recherche, nous avons identifié et défini les thèmes principaux liés à notre objet d'étude, à savoir «improvisation», «musique classique» et «experts».

\section{Improvisation}

En situant l'improvisation au regard de la composition, Kingscott et Durrant (2010) remarquent que la première se distingue de la seconde par le fait qu'elle prend forme à travers les interactions sociales et qu'elle se manifeste sous la forme d'un évènement ponctuel plutôt que sous celle d'un opus immuable dans le temps. Dans le cadre de la présente recherche, nous définissons l'improvisation comme étant: «une réalisation instrumentale ou vocale où le musicien génère du matériau musical en temps réel, tout en étant capable d'anticiper la conséquence sonore de ses actions sur la base de ses expériences passées» (Dubé et Després 2012, p. 144). 


\section{Musique classique}

Alors qu'un auditeur expérimenté n'éprouvera probablement aucune difficulté à distinguer la musique "classique» des autres genres musicaux, formuler une définition consensuelle de la musique classique s'avère une entreprise complexe. Dans le cadre de la présente recherche, les experts consultés ont reçu une formation en musique classique de niveau baccalauréat (ou équivalent) (voir section Participants). Ainsi, nous avons évalué qu'ils étaient aptes à juger de l'adéquation de leur pratique en improvisation avec le genre "musique classique »; lors du recrutement, les participants étaient appelés à juger par euxmêmes à savoir si le type d'improvisation qu'ils pratiquent correspond ou non à ce genre.

\section{Experts}

Étudier le comportement des experts permet une meilleure compréhension des limites de capacités humaines et des conditions d'apprentissage optimal (Ericsson et Charness 1994). Un expert peut être défini comme:

The distinguished or brilliant journeyman, highly regarded by peers, whose judgments are uncommonly accurate and reliable, whose performance shows consummate skill and economy of effort, and who can deal effectively with certain types of rare or "tough" cases. Also, an expert is one who has special skills or knowledge derived from extensive experience with subdomains. (Chi 2006, p. 122)

Selon cette définition, l'expert n'a pas nécessairement atteint le stade du «maitre», qui est un expert parmi les experts (Chi 2006), mais il se démarque toutefois dans son domaine d'expertise. De façon générale, les experts se distinguent des novices par la taille et la flexibilité de leur base de connaissances qui leur permettent d'anticiper et de réagir plus adéquatement, et de mettre en œuvre des stratégies qui sont mieux adaptées, aux problèmes qu'ils rencontrent (Lemaire et Siegler 1995). Dans le contexte de l'improvisation musicale, les experts possèdent également des compétences qui sont spécifiques à leur domaine telles que: (1) la capacité d'entendre intérieurement leurs idées musicales avant de les jouer; (2) les habiletés techniques nécessaires pour réaliser leurs intentions musicales; (3) une connaissance des conventions stylistiques du ou des langages avec lesquels ils sont familiarisés et (4) une conscience aiguë du lien entre la structure de la musique et son pouvoir communicationnel (Kratus 1991). Les critères qui ont été utilisés pour déterminer l'expertise dans le contexte spécifique de la présente recherche sont explicités dans la section suivante.

\section{MÉTHODE}

Notre recherche combine des données qui proviennent de deux collectes de données distinctes, lesquelles s'inscrivent dans un projet de recherche 
tripartite portant sur l'apprentissage et la production de l'improvisation musicale classique 5 .

\section{Participants}

Des improvisateurs experts reconnus et des médias spécialisés ont été consultés afin de dresser une liste de participants potentiels pour la recherche. Pour participer, les musiciens devaient appartenir à la catégorie des experts en improvisation musicale classique ou à celle des enseignants experts en improvisation musicale classique au niveau postsecondaire. Des courriels d'invitation personnels ont été envoyés à tous les musiciens qui semblaient satisfaire aux critères d'échantillonnage respectifs de l'une ou l'autre de ces catégories. L'admissibilité du participant était ensuite validée par le chercheur. Pour être reconnu comme improvisateur expert en musique classique, le participant avait:

1. atteint un niveau d'expertise en improvisation reconnu par ses pairs;

2. complété minimalement une formation de niveau baccalauréat (ou équivalent) en musique classique;

3. improvisé sur au moins un album professionnel; et

4. improvisé dans des salles de concert reconnues.

Pour être reconnu comme étant expert en enseignement de l'improvisation musicale classique au niveau postsecondaire, le participant avait:

1. atteint un niveau d'expertise en enseignement de l'improvisation reconnu par ses pairs;

2. complété minimalement une formation de niveau baccalauréat (ou équivalent) en musique classique;

3. enseigné l'improvisation dans un programme de musique classique de niveau postsecondaire dans un établissement reconnu.

Les deux catégories n'étant pas exclusives, un même participant pouvait être reconnu comme étant à la fois expert en improvisation musicale classique et enseignant expert en improvisation musicale classique au niveau postsecondaire, à condition de répondre à l'ensemble des critères précédents.

Au total, 15 participants ont participé à l'une ou l'autre des deux phases de la recherche: 10 participants pour la phase 1, et 5 participants pour la phase 2. Parmi ces 15 participants, 13 ont été identifiés comme étant experts en improvisation musicale classique et 11 comme enseignants experts en improvisation musicale classique. Sept participants étaient originaires du Canada, cinq de la France, un d'Israël, un de l'Allemagne et un du Mexique. Un seul participant, bien qu'identifié comme étant expert en improvisation musicale classique, ne répondait pas au critère 3 lié à cette catégorie. Nous avons néanmoins choisi de le retenir dans notre échantillonnage, en raison de son expérience en improvisation sur la scène internationale. Une fois le recrutement et l'explication des

5 Les résultats des deux phases précédentes seront publiées dans : Després, J. P., Burnard, P., Dubé, F. et Stévance, S. (accepté). Expert improvisers in Western classical music learning pathways. Thinking Skills and Creativity. Després, J. P., Burnard, P., Dubé, F. et Stévance, S. (soumis). Expert Western classical music improvisers' strategies. 
considérations éthiques complétées, nous avons envoyé au participant un bref questionnaire afin de collecter, avant la tenue de l'entrevue, ses données démographiques et autres informations relatives à son expérience en improvisation (voir Tableau 1). Des noms fictifs ont été utilisés ici pour assurer l'anonymat des participants. 
Tableau 1. Données démographiques et expertises des participants.

\begin{tabular}{|c|c|c|c|c|c|}
\hline & Nom & Sexe & $\begin{array}{l}\text { Instrument } \\
\text { principal }\end{array}$ & $\begin{array}{l}\text { Improvisateur } \\
\text { expert }\end{array}$ & $\begin{array}{c}\text { Expert en } \\
\text { enseignement } \\
\text { de l'improvisation }\end{array}$ \\
\hline \multirow{10}{*}{$\begin{array}{l}\bar{d} \\
\infty \\
0 \\
\overline{0} \\
\bar{\alpha}\end{array}$} & David & M & Guitare & & $\mathrm{x}$ \\
\hline & Philippe & M & Piano & $\mathrm{x}$ & $\mathrm{x}$ \\
\hline & Sébastien & M & Guitare & $\mathrm{x}$ & \\
\hline & Thierry & M & Piano & $\mathrm{x}$ & \\
\hline & Patricia & $\mathrm{F}$ & Piano & $\mathrm{x}$ & $\mathrm{x}$ \\
\hline & Lise & $\mathrm{F}$ & Piano & & $\mathrm{x}$ \\
\hline & Isaac & M & Piano & $\mathrm{x}$ & $\mathrm{x}$ \\
\hline & Franck & M & Violon & $\mathrm{x}$ & $\mathrm{x}$ \\
\hline & Sophie & $\mathrm{F}$ & Contrebasse & $\mathrm{x}$ & $\mathrm{x}$ \\
\hline & Daniel & M & Orgue et piano & $\mathrm{x}$ & $\mathrm{x}$ \\
\hline \multirow{5}{*}{$\begin{array}{l}N \\
\vdots \\
0 \\
\mathbb{D} \\
\frac{5}{0} \\
\frac{5}{0}\end{array}$} & David & M & Orgue & $x$ & \\
\hline & Stéphane & M & Contrebasse & $\mathrm{x}$ & $\mathrm{x}$ \\
\hline & Raúl & M & Guitare & $\mathrm{x}$ & $\mathrm{x}$ \\
\hline & Jimmy & M & Guitare & $\mathrm{x}$ & \\
\hline & Line & $\mathrm{F}$ & Orgue & $\mathrm{x}$ & $\mathrm{x}$ \\
\hline
\end{tabular}

\section{Collectes de données}

Au total, nous avons mené 15 interviews, 10 par visioconférence et 5 en personne. Lors de la première phase de collecte, des experts en improvisation ou en enseignement de l'improvisation musicale classique au niveau postsecondaire ont été interviewés par vidéoconférence. L’entrevue par vidéoconférence est une méthode de collecte de données qui, bien qu'elle soit toujours peu usitée dans le domaine de la recherche qualitative, présente des qualités qui surpassent généralement ses limites et inconvénients potentiels (Nehls, Smith et Schneider 2015). Dans le cas de la présente étude, la vidéoconférence nous a permis d'augmenter considérablement l'accessibilité de notre recherche et, par le fait même, le nombre de participants, tout en nous offrant la possibilité de constituer un échantillon international. L’application Skype a été utilisée pour réaliser la vidéoconférence, en combinaison avec l'application Call Recorder pour Skype, qui permet d'enregistrer les vidéoconférences. Lors de ces entretiens, le chercheur a d'abord invité le participant à décrire son parcours d'apprentissage de l'improvisation, puis à discuter des stratégies qu'il met en œuvre lors de l'improvisation. Finalement, le chercheur a invité le musicien à partager les approches et stratégies qu'il utilise lorsqu'il enseigne l'improvisation, lorsque ce dernier était également reconnu comme étant enseignant expert en improvisation musicale classique au niveau postsecondaire.

Lors de la seconde phase de collecte de données, des experts en improvisation musicale classique ont participé à des rencontres individuelles en personne avec le chercheur principal. Lors de ces rencontres, le chercheur a d'abord invité le participant à partager son parcours d'apprentissage de l'improvisation. 
Par la suite, le chercheur a invité le participant à improviser. Finalement, le chercheur a invité le musicien à partager les approches et stratégies qu'il utilise lorsqu'il enseigne l'improvisation, lorsque ce dernier était également reconnu comme étant enseignant expert en improvisation musicale classique au niveau postsecondaire. La rencontre a été intégralement filmée, afin de recueillir les propos du musicien en vue de les analyser par la suite.

\section{Analyse des données}

Nous avons effectué les transcriptions des entrevues à l'aide du logiciel Transcriptions de David Haselberger. Par la suite, nous avons réalisé une analyse thématique des entrevues (Paillé et Mucchielli 2012) en adoptant une approche inductive, où les thèmes émergent des matériaux analysés. Tous les énoncés des participants liés au parcours d'apprentissage ou aux approches d'enseignement de l'improvisation musicale ont été extraits et codés. En construisant progressivement notre arborescence de codage, nous avons formulé des définitions opérationnelles pour chaque nouvelle catégorie créée et affiné notre catégorisation, en fusionnant et en renommant les catégories créées au besoin.

Après avoir terminé le codage, l'accord interjuge a été vérifié par un chercheur senior (Doctorat en musique) familiarisé avec la recherche qualitative et le codage de données. L'évaluateur externe a ainsi codé indépendamment $10 \%$ des données, en utilisant un modèle ouvert. Ensuite, nous avons discuté des résultats divergents et retravaillé nos définitions opérationnelles de sorte que toutes les catégories atteignent un coefficient de Kappa supérieur à o,80.

\section{RÉSULTATS}

Plus d'une cinquantaine de recommandations pédagogiques et de stratégies d'enseignement-apprentissage de l'improvisation ont été organisées en deux catégories:

1. Compétences à développer chez l'apprenant: qualités ou aptitudes qui influenceraient favorablement le développement des habiletés d'improvisation;

2. Approches d'enseignement-apprentissage: propositions d'activités concrètes visant à développer les habiletés d'improvisation de l'apprenant.

Dans les prochaines lignes, nous détaillerons les composantes appartenant à chacune de ces catégories.

\section{Compétences à développer chez l'apprenant}

Tout au long des entrevues, les participants ont mentionné 18 compétences (listées et définies dans le Tableau 2 - Compétences à développer chez l'apprenant) qui, à leur avis, devaient être développées par l'apprenant improvisateur. Nous avons regroupé ces compétences en trois grandes catégories: (1) musicales et théoriques; (2) synchrones; et (3) autodidactiques. 


\section{2) Musicales et théoriques}

Plusieurs compétences liées à des habiletés musicales ou à des connaissances théoriques sont essentielles au développement de l'habileté en improvisation. Dans le cadre de cette recherche, onze compétences musicales et théoriques ont été recensées: la connaissance de l'harmonie, des gammes et des arpèges, l'audition intérieure, la superposition thématique, la transposition, le repiquage, la customisation, la reconnaissance des connaissances antérieures, l'application des connaissances antérieures, l'intégration de l'approche d'analyse jazz aux connaissances antérieures et la direction d'orchestre.

Au sujet de l'audition intérieure, Daniel partage:

C'est très important pour improviser: parce que ce que j'entends dans la tête, il n'y a plus qu'à le jouer. Alors, une fugue, ça ne se travaille pas à la guitare. Une fugue, ça se travaille dans la tête. Et si vous entendez la fugue dans la tête, si vous la rêvez suffisamment longtemps vous allez l'entendre ... il n'y a plus qu'à la jouer.

Au sujet de la transposition, Stéphane rigole en affirmant: "C’est toujours bon de pouvoir jouer dans tous les tons ... je pense qu'il y en a un qui a compris ça il y a 300 ans, il a écrit deux livres dans tous les tons, tu sais». La capacité d'intégration de l'approche d'analyse jazz aux connaissances antérieures permettrait notamment, selon Stéphane, d’accélérer la capacité de transposition de l'improvisateur:

Premier exercice de base d'improvisation: je leur donne une phrase, j'en écris quelques-unes et je leur dis de les jouer dans tout le cycle des quintes, dans toutes les tonalités. Il faut apprendre à penser plus par chiffre que par note.

Franck note, chez les musiciens classiques auxquels il enseigne, un important décalage entre les connaissances théoriques et la capacité à analyser le répertoire sur le vif. C'est pourquoi il insiste sur l'importance de développer leur capacité de reconnaissance de ses connaissances antérieures dans le répertoire. Par exemple, l'apprenant qui aiguise ses capacités d'analyse et qui apprend à identifier les modes qu'il connaît en théorie "peut analyser Brahms et voir: c'est la gamme myxolydienne qu'il utilise ici» (Franck). Toujours selon Franck, parallèlement à ce processus, l'apprenant devra également apprendre à appliquer ses connaissances antérieures lors de l'improvisation. En effet, il y a un clivage important chez plusieurs musiciens classiques entre les connaissances théoriques et la capacité à appliquer ces connaissances à l'instrument. C'est pourquoi «quand je parle d'improviser totalement, je commence avec le bagage [que l'élève] connaît déjà : je débute avec des gammes majeures, mineures, la gamme myxolydienne ...» (Franck).

\section{3) Synchrones}

Les compétences synchrones sont liées au fait que l'improvisation se déroule en temps réel et qu'elle exige du musicien qu'il se plonge dans un état particulier 
afin de pouvoir réagir aux occurrences et créer de nouvelles idées musicales en temps réel. Les six compétences synchrones que nous avons recensées sont: la "présence», la prise de risques, la capacité d'adaptation, l'ouverture, la concertation entre l'instinct et le savoir-faire et l'accueil de la "fausse» note.

L'état de présence a été dénommé de différentes façons par les participants: jouer avec «instinct» (Isaac), être «présent» (Daniel) se mettre «dans l'espace sacré» (Daniel), "être dans le moment présent et pas juste "utiliser tes doigts comme des doigts"» (Jimmy), lors de l'improvisation. Par ailleurs, pour Issac, la prise de risque est une composante nécessaire de l'improvisation:

Improviser, ça veut dire unifier ou fusionner l'acte de l'interprétation et de la création du matériel. Donc, l'exécution et la création se réunissent, deviennent un en temps réel. Mais on peut composer spontanément une composition qui va avoir peu d'improvisation ou d'esprit d'improvisation, avec très peu de prise de risques par exemple. Ce qui, pour moi, fait partie d'une vraie improvisation: d'inclure sincèrement l'inconnu ou l'imprévu.

Ensuite, lorsque nous l'avons interrogé sur sa disposition éventuelle d'outils ou de stratégies pour développer la capacité de prise de risque, il nous a répondu:

[...] non, il n'y a pas de stratégies sauf de ne pas oublier le fait que quand nous vivons notre vie, nous sommes en face l'imprévu tout le temps. Donc c'est quelque chose qui fait partie de vivre une vie.

Alors que les compétences synchrones semblent être intrinsèquement liées à l'improvisation - en raison du fait que cette dernière est créée en temps réel -, les compétences autodidactiques seraient transversales aux autres compétences musicales.

\section{4) Autodidactiques}

Les compétences autodidactiques seraient d'autant plus importantes pour apprendre l'improvisation musicale classique que, dans le contexte actuel, cette dernière occupe un rôle marginal au sein du curriculum. En effet, l'improvisation étant très peu enseignée dans les institutions formelles relativement aux autres matières, le musicien classique devra apprendre à apprendre par luimême pour développer cette compétence. Deux compétences autodidactiques ont été proposées par les participants de la présente recherche, soit la motivation intrinsèque et les habiletés métacognitives. Les compétences métacognitives correspondent à la capacité que possède un individu à observer et à agir sur ses propres processus mentaux (Plucker, Runco et Hegarty 2011). Ces compétences permettent notamment au musicien de développer ses habiletés en improvisation, sans nécessairement recevoir l'aide ou l'appui d'un guide extérieur. Par exemple, pour Patricia, l'objectif de l'enseignant en musique devrait être de développer la motivation intrinsèque de l'apprenant, ce qui peut être fait, notamment, en éveillant sa curiosité:

[...] [souvent, les étudiants me demandent:] «Oui, mais comment on fait pour improviser?», «Oui mais, c'est quoi la méthode?» enfin, toujours 
à demander, au lieu de chercher par soi-même. Voilà, c'est peut-être là le souci: comment inciter les gens à avoir cette curiosité finalement, avant toutes choses, avant de leur donner le truc tout cru. [...] Oui, apprendre à apprendre c'est ça. Être curieux et être d'accord pour être déstabilisé à chaque instant. C'est ça finalement, pour apprendre, il faut vraiment oser mettre un pas devant et accepter l'inconnu, avoir le désir plus fort que la peur d'avancer. [...] Je trouve que l'improvisation, pour ça, c'est idéal. C'est idéal parce qu'il y a cet inconnu, qui est encore plus fort.

Pour leur part, les habiletés métacognitives correspondent à la capacité que possède un individu à observer et à agir sur ses propres processus mentaux (Plucker, Runco et Hegarty 2011); elles se manifestent notamment par les capacités de monitoring et d'auto-évaluation réflexive. Les compétences musicales et théoriques, synchrones et autodidactiques décrites par les participants sont celles qu'ils ont développées eux-mêmes lors de leur apprentissage ou qu'ils cherchent à développer chez leurs élèves.

Tableau 2. Compétences à développer chez l'apprenant improvisateur

Catégorie

Compétence

Musicales et théoriques

Connaissance de l'harmonie, des gammes et des arpèges

Audition intérieure

Superposition thématique

Transposition

Repiquage

Customisation

Reconnaissance de ses connaissances antérieures

Application de ses connaissances antérieures

Intégration de l'approche d'analyse jazz aux connaissances antérieures

Direction d'orchestre

\section{Synchrones}

«Présence»

Prise de risques

Capacité d'adaptation
Définition

\section{Compétences liées aux connaissances et habiletés instrumen-} tales et théoriques.

Connaître, à la fois sur le plan théorique et pratique (instrumental), l'harmonie classique ainsi que plusieurs gammes et arpèges.

Entendre intérieurement les sons avant de les jouer.

Ajouter un thème au-dessus ou en dessous de n'importe quel passage musical, mélodique ou harmonique (pour les instruments polyphoniques).

Transposer des idées musicales (motifs, phrases et thèmes) dans tous les tons.

Jouer des pièces connues à l'oreille et les transposer.

Personnaliser les idées musicales tirées du répertoire, pour ultimement les intégrer à l'improvisation.

Reconnaître, dans le répertoire et lors de l'improvisation, les éléments théoriques connus.

Mettre en pratique ses connaissances théoriques et instrumentales lors de l'improvisation.

Superposer la pensée analytique jazz à l'analyse et au répertoire classiques. Par exemple, en pensant au nom des degrés plutôt qu'à celui des notes.

Diriger un orchestre (ou même un petit ensemble ou un soliste).

\section{Compétences liées à la capacité de créer et de réagir en temps} réel.

Mettre de côté les attentes et les jugements et être dans le moment présent lors de l'improvisation.

Oser prendre des risques lors de l'improvisation et accepter toutes les occasions de faire de la musique improvisée.

Développer une technique instrumentale polyvalente, permettant de s'adapter à différentes situations et de pouvoir sortir de ses habitudes. 
Tableau 2 (suite)

Catégorie

Compétence
Définition

\begin{tabular}{ll}
\hline $\begin{array}{l}\text { Ouverture } \\
\begin{array}{l}\text { Concertation entre l'instinct } \\
\text { et le savoir-faire } \\
\text { L'accueil de la «fausse» } \\
\text { note }\end{array}\end{array}$ & $\begin{array}{l}\text { Élargir ses possibilités musicales, en acceptant un vaste spectre } \\
\text { d'avenues musicales. }\end{array}$ \\
\hline Autodidactiques & $\begin{array}{l}\text { Intégrer les notes inattendues à l'improvisation en leur donnant un } \\
\text { sens musical et en utilisant leur potentiel créatif. }\end{array}$ \\
Motivation intrinsèque & Compétences liées à la capacité d'apprendre par soi-même. \\
& $\begin{array}{l}\text { Développer un intérêt intrinsèque pour l'apprentissage et le } \\
\text { développement d'habiletés d'improvisation. }\end{array}$ \\
Habiletés métacognitives & $\begin{array}{l}\text { Prendre conscience de son propre processus d'apprentissage : } \\
\text { s'autoévaluer, planifier ses «pratiques » et documenter ses progrès. }\end{array}$ \\
\hline
\end{tabular}

\section{Approches d'enseignement-apprentissage}

Les participants ont également mentionné des approches d'enseignement-apprentissage qui permettraient de développer l'habileté en improvisation de l'apprenant ainsi que certaines des compétences mentionnées dans la section précédente. Ces approches découlent de leur propre expérience d'apprentissage de l'improvisation ou de leur pratique d'enseignement de l'improvisation.

\section{5) Fondamentales}

Nous avons identifié deux approches préparatoires à l'improvisation qui, sans être des activités d'improvisation à proprement parler, permettent au musicien de développer son aptitude en improvisation par un processus d'enculturation et par le développement de sa base de connaissances: écouter les grands et construire sa banque d'idées. En ce qui a trait à l'importance d'écouter les grands, Stéphane affirme "Ça peut être le meilleur enseignement, je pense». Au sujet du processus de construction de sa banque d'idées, Sébastien exprime:

[Repiquer des motifs à l'oreille m'amenait], en essayant de les apprendre, à les transformer automatiquement, parce que parfois je ne repiquais pas exactement la bonne chose ou je tombais sur quelque chose d'autre. Donc là je recréais des lignes, puis je me faisais carrément des cahiers de lignes.

Isaac offre des propos similaires: «[...] plonger dans les textes et tirer des éléments caractéristiques de maîtres de certaines périodes, même de certaines œuvres et de les personnaliser, de les rendre customized». Alors que ces deux approches fondamentales ne sont pas directement liées à des activités d'improvisation, les autres approches que nous avons identifiées le sont; il s'agit de stratégies qui peuvent être mises en œuvre aussi bien lors de répétitions que lors de prestations improvisées. 


\section{6) Planificatrices}

Les approches planificatrices sont des décisions qui sont prises avant de jouer et qui permettent de déterminer à l'avance certaines caractéristiques de l'improvisation. Deux approches planificatrices sont ressorties des entrevues: esquisser l'improvisation et poser des contraintes. Lise donne un exemple concret de comment on peut esquisser l'improvisation, tout en affirmant que cette approche favorise une plus grande cohérence de l'improvisation.

[...] en fait, n'importe quelle improvisation part d'une idée: soit un motif, un rythme ... Alors on part de ça et puis je dis souvent [aux élèves]: «Donnez-vous un 5-10 minutes, juste pour établir au moins la structure, les grandes lignes, le canevas: [par exemple] une personne commence, après ça l'autre s'ajoute, la $3^{\mathrm{e}} \ldots$ on forme quelque chose d'assez dynamique, en trois temps. Après ça, la forme ... le $\mathrm{B}$ arrive ... alors le $\mathrm{B}$ ça va être quoi?» Juste de délimiter: «Ok, ça va être un duo pendant que la $3^{\mathrm{e}}$ personne va simplement supporter. Après ça on va revenir tout le monde ensemble pour la partie A». Juste établir ça, et souvent c'est suffisant pour que l'improvisation s'organise.

Un grand nombre de contraintes peuvent être posées par les musiciens ou par l'enseignant avant l'improvisation, par exemple:

1. Courbe de dynamiques préétablie (Patricia et Franck): «Peu importe ce que l'on fait, il faut faire un crescendo qui commence du plus doux et qui finisse très fort. Et quand on arrive à notre maximum de forte, la musique s'arrête» (Patricia);

2. Polytempi: «[Il faut que] chacun ait un tempo différent et que, quoi qu'il arrive, on le garde ${ }^{6} . »$ (Patricia);

3. Se fondre dans l'autre: "Rentrer dans le timbre de l'autre. Par exemple, la contrebasse va proposer un timbre particulier avec l'archet, et comment les deux autres instruments peuvent se fondre avec ça et puis se passer le truc.» (Patricia);

4. Casser: «Il y a deux [musiciens] qui sont vraiment en symbiose et le $3^{\mathrm{e}}$ vient casser systématiquement» (Patricia);

5. Tours de solo: «Travailler sur la notion de solo. Les deux autres [musiciens] accompagnent et puis se passent le solo» (Patricia);

6. Jeux de question-réponse (Patricia);

7. Techniques contrapuntiques (par exemple, l'imitation et le canon) (Patricia), etc.

Qu'il s'agisse de déterminer la structure formelle de l'improvisation ou d'autres de ces caractéristiques avant de commencer à jouer, les stratégies planificatrices servent de guide aux musiciens improvisateurs et permettent, dans l'éventualité d'une improvisation de groupe, de créer un cadre partagé par tous les musiciens avant de commencer à jouer.

6 Note du chercheur : on pourrait commencer l'exercice avec des métriques différentes, avant de travailler avec des tempi différents. 


\section{7) Sonores}

Les participants ont proposé deux approches sonores qui sont respectivement centrées sur le timbre de l'instrument (travailler la laideur et la beauté du son) et sur la texture d'une section de l'improvisation (focaliser sur les textures sonores) plutôt que sur la hauteur des sons. Pourquoi travailler la beauté du son, en passant d'abord par sa laideur? Parce que, selon Daniel: «Qui ne sait pas faire un son laid, ne connaît pas la limite de la laideur et donc [il] est toujours en deçà, et ce n'est pas très beau ". Ainsi, en osant toucher les frontières sonores de son instrument, l'apprenant pourrait apprendre à dépasser ses peurs de produire un son inesthétique et ainsi pouvoir améliorer le contrôle qu'il exerce sur sa sonorité. Pour Franck, focaliser sur les textures sonores veut dire: «faire de la sculpture sonore, du soundpainting 7 comme on appelle ça aussi». L'utilisation des approches sonores montre que le rythme et même la hauteur des sons peuvent occuper une place secondaire dans l'attention du musicien improvisateur pendant l'improvisation ou un segment de l'improvisation.

\section{8) Conceptuelles}

Six approches conceptuelles d'enseignement-apprentissage de l'improvisation sont ressorties des entrevues que nous avons menées: faire de l'improvisation rythmique pure, jouer en déplaçant les accents, faire de l'improvisation mélodique, utiliser la gamme chromatique, s'ancrer sur tonique-dominante et faire appel aux techniques contrapuntiques. Jouer en déplaçant les accents, lors de l'interprétation de pièces classiques ou lors de l'improvisation, procure à l'apprenant, selon Franck, «un contrôle sur le tempo qui est beaucoup plus précis». Pour Sébastien, utiliser la gamme chromatique, ou en venir à " considérer que c'est la gamme chromatique la gamme de base» lors de l'improvisation est une des étapes avancées de l'apprentissage de l'improvisation. Pour Philippe, s'ancrer sur tonique-dominante est une approche qui permet, presque immanquablement, de produire une improvisation «qui se tient»:

Donc si, au piano, tu joues [à la main gauche] une note entre le fa et le $d o$, tonale dominante, tu vas la faire en pulsation, simplement. À droite, tu vas jouer tranquillement quelques notes, n'importe lesquelles, mais tu finis ta phrase musicale, de 4, 5, 6 notes maximum, toujours soit sur $f a$, soit sur $d o$. Et tu verras, tout se résout.

Les approches conceptuelles sont principalement orientées autour du rythme ou de la hauteur des sons, alors que les approches structurelles concernent des segments musicaux plus longs.

\section{9) Structurelles}

Quatre approches structurelles sont ressorties de l'analyse des entrevues, la première (utiliser sa boitte d'idées) se situe sur le plan micro-structurel du motif

7 Habituellement, le soundpainting constitue plutôt un «universal multidisciplinary live composing sign language for musicians, actors, dancers, and visual Artists» (2016) créé par Walter Thompson en 1974 . 
ou de la phrase, tandis que les trois autres (improviser sur une structure harmonique, faire de l'improvisation formelle et ériger la structure en temps réel) déterminent plutôt les aspects macro-structurels de l'improvisation. Lorsque Franck propose à ses étudiants de faire de l'improvisation formelle, il peut, par exemple, leur demander d'improviser un scherzo - même si l'improvisation est abstraite et pas nécessairement tonale - ou encore d'improviser en respectant la forme ABACABA - ce qui les oblige à se concentrer pour mémoriser les idées musicales qu'ils improvisent, afin de pouvoir les rappeler, de manière intégrale ou permutée, lors du retour de la section A ou B. Alors que la forme peut être prédéfinie avant de commencer à improviser, il est également possible d'ériger la structure en temps réel, un processus que Jimmy décrit comme suit:

Mais j'essaie vraiment, quand j'enseigne l'improvisation ... [de travailler] le plus possible les effets de la relation entre le matériel, la relation entre les deux partenaires, il faut s'écouter, il faut ériger la structure en temps réel.

Ainsi, en plus de déterminer les hauteurs des sons ainsi que la configuration rythmique des notes jouées, les improvisateurs experts mettent en œuvre diverses approches structurelles afin d'organiser l'ensemble de l'improvisation en un tout cohérent.

\section{0) Stylistiques}

Parmi les approches stylistiques, on retrouve faire de l'improvisation «sans style», faire de l'improvisation générative, improviser dans le style de ... et ornementer. Lorsqu'il enseigne aux débutants, Jimmy cherche à faire de l'improvisation «sans style», tout en ajoutant, paradoxalement, que «c'est impossible»:

Normalement quand je donne des ateliers, on parle d'improvisation sans style. On ne joue pas du blues, on ne joue pas du jazz, on ne joue même pas du free. On essaie juste ... je sais que c'est impossible de jouer, entre guillemets, sans références culturelles, parce que tout vient d'une culture quelque part.

Patricia décrit comment elle procède pour faire de l'improvisation générative ${ }^{8}$ avec les apprenants:

C'est un travail sur le timbre, sur l'écoute. Systématiquement, on fait un travail corporel d'abord, pour être vraiment au fond de soi, bien en soi, être bien détendu, mais aussi être bien concentré, être bien profond, et après on installe des petits jeux très simples de dialogue, on va dire physique. Après, on essaie de transposer ce qu'on a trouvé physiquement avec l'instrument.

8 «Par le terme improvisation générative, on désigne une forme d'improvisation libre, basée sur des principes d'écoute et d'invention musicale instantanée. Elle n'obéit pas à un style ou un idiome musical, toutefois les questions de mémoire individuelle et collective sont présentes et les liens avec les musiques traditionnelles, le jazz et la musique contemporaine sont nombreux. » http://revues. mshparisnord.org/filigrane/index.php? $\mathrm{id}=395$ 
Les approches stylistiques peuvent aussi bien être utilisées pour établir les règles générales d'organisation du discours musical que pour inciter le musicien à transcender ses habitudes et à demeurer dans le moment présent lors de l'improvisation.

\section{1) Contextuelles}

Trois approches contextuelles sont ressorties des entrevues que nous avons menées: faire de l'improvisation interdisciplinaire, cultiver le sentiment d'urgence et jouer avec des musiciens plus expérimentés. Patricia partage une activité d'improvisation interdisciplinaire qu'elle a mise en œuvre avec ses élèves:

Récemment on avait 3-4 danseuses qui nous ont proposé des thèmes: le végétal, le minéral, l’animal. Elles avaient un peu cherché déjà des choses. Le professeur de danse est venu avec elles et on a travaillé toute une journée sur: comment est-ce qu'on peut faire de la musique sur cette proposition de thème et donc de mouvement?

Patricia a également témoigné de l'apport de l'approche qui consiste à cultiver le sentiment d'urgence lors des activités pédagogiques d'improvisation:

On avait un but: à la fin de la journée, à 5 heures du soir, il fallait faire une prestation, avec éclairage, enfin une mise en situation, une mise en valeur avec un public et tout. Et ça fonctionne très bien, parce qu'il y a une espèce d'urgence.

Les différentes approches contextuelles que nous avons identifiées ont comme effet escompté de stimuler la créativité du musicien, soit en le plaçant dans une situation de création inhabituelle, soit en lui imposant un «stress» créateur positif.

Tableau 3. Approches d'enseignement-apprentissage de l'improvisation

\begin{tabular}{ll}
\hline \multicolumn{1}{c}{$\begin{array}{c}\text { Catégorie } \\
\text { Approches }\end{array}$} & \multicolumn{1}{c}{ Description } \\
\hline Fondamentales & $\begin{array}{l}\text { Approches effectuées en marge de } \\
\text { l'improvisation. } \\
\text { Écouter les grands musiciens improvisateurs } \\
\text { pour assimiler leur langage. }\end{array}$ \\
Construire sa banque d'idées & $\begin{array}{l}\text { S'approprier, de manière exacte ou libre, des } \\
\text { idées musicales provenant du répertoire, en les } \\
\text { travaillant à l'instrument et les consignant lors de }\end{array}$ \\
\hline Planificatrices & sa pratique » personnelle. \\
Esquisser l'improvisation & $\begin{array}{l}\text { Approches qui impliquent une planification } \\
\text { de l'improvisation. }\end{array}$ \\
Poser des contraintes & $\begin{array}{l}\text { Préparer un canevas de l'improvisation avant de } \\
\text { commencer à jouer. }\end{array}$ \\
& $\begin{array}{l}\text { Déterminer les règles qui régissent l'improvisa- } \\
\text { tion avant de commencer à jouer. }\end{array}$
\end{tabular}


Tableau 3 (suite)

Catégorie

Approches

Description

\section{Sonores}

Travailler la laideur et la beauté du son

Focaliser sur les textures sonores

\section{Conceptuelles}

Faire de l'improvisation rythmique pure

Jouer en déplaçant des accents

Faire de l'improvisation mélodique

Utiliser la gamme chromatique

S'ancrer sur tonique-dominante

Faire appel aux techniques contrapuntiques

\section{Structurelles}

Utiliser sa boîte d'idées

Improviser sur une structure harmonique

Faire de l'improvisation formelle

Ériger la structure en temps réel

\section{Stylistiques}

Faire de l'improvisation «sans style» prédéfini

Faire de l'improvisation générative

Improviser dans le style de...

\section{Approches liées à la qualité sonore de l'improvisation.}

Travailler la beauté du son, en passant d'abord par sa laideur pour mieux connaître les limites et son instrument.

Improviser en se concentrant sur la texture sonore (plutôt que sur la hauteur des notes).

\section{Approches centrées sur l'utilisation de con-} cepts musicaux lors de l'improvisation.

Improviser une partie purement rythmique, en utilisant, par exemple, des percussions corporelles, des instruments de percussion ou des sons sans hauteur à l'instrument.

Jouer du répertoire ou des improvisations classiques avec des appuis «jazz», sur les temps 2 et 4 , pour améliorer son contrôle rythmique.

Improviser une ligne mélodique, par exemple, sur une pédale, un ostinato, une métrique ou une structure formelle.

Développer l'improvisation à partir de la gamme chromatique, pour sortir de ses patrons tonals habituels (gammes, modes et arpèges, le cas échéant).

Improviser en achevant chaque phrase sur la tonique ou la dominante.

Utiliser des techniques contrapuntiques lors de l'improvisation (imitation exacte ou non, canon, ajout de voix contrapuntiques, etc.).

\section{Approches liées aux motifs, aux phrases ou} à la forme de l'improvisation.

Utiliser, modifier ou transposer des éléments tirés de la banque d'idées lors de l'improvisation.

Improviser à partir d'un court enchaînement harmonique tiré du répertoire, pour créer un cadre rassurant.

Improviser en respectant une structure formelle préétablie.

Improviser la structure formelle même de l'improvisation.

\section{Approches centrées sur les règles d'un langage musical donné.}

Improviser sans limites stylistiques, pour laisser jaillir la musique sans restriction.

Faire de l'improvisation "générative », c'est-àdire où un son en amène un autre.

Improviser dans le style d'un compositeur, d'une œuvre ou d'une époque. 
Tableau 3 (suite)

\begin{tabular}{ll}
\hline \multicolumn{1}{c}{$\begin{array}{c}\text { Catégorie } \\
\text { Approches }\end{array}$} & \multicolumn{1}{c}{ Description } \\
\hline Ornementer & $\begin{array}{l}\text { Rejouer plusieurs fois une même pièce en vari- } \\
\text { ant les ornements à chaque fois. }\end{array}$ \\
\hline Contextuelles & $\begin{array}{l}\text { Approches centrées sur le contexte dans } \\
\text { lequel se déroule l'improvisation. }\end{array}$ \\
Faire de l'improvisation interdisciplinaire & $\begin{array}{l}\text { Improviser en collaboration avec d'autres arts, } \\
\text { par exemple la danse, le théâtre ou les arts } \\
\text { visuels. }\end{array}$ \\
Cultiver le sentiment d'urgence & $\begin{array}{l}\text { Préparer une prestation improvisée qui sera } \\
\text { présentée dans un court délai (par exemple, la } \\
\text { journée même) afin de stimuler le processus } \\
\text { créatif. } \\
\text { Jouer avec des musiciens plus expérimentés }\end{array}$ \\
& $\begin{array}{l}\text { Partager la scène ou répéter avec des musiciens } \\
\text { plus expérimentés que soi. }\end{array}$ \\
\hline
\end{tabular}

\section{Discussion}

Nous avons organisé nos données de recherches en deux composantes: 18 compétences à développer chez l'apprenant, regroupées en trois catégories, et 23 approches d'enseignement-apprentissage, regroupées en sept catégories. Afin de répondre à notre premier objectif, «recenser les recommandations pédagogiques et stratégies d'enseignement-apprentissage de musiciens et de pédagogues experts en improvisation musicale classique", nous allons d'abord présenter les contributions de notre recherche par rapport à la littérature existante. Ensuite, pour atteindre notre deuxième objectif, «structurer les éléments recensés afin de formuler une proposition pédagogique cohérente», nous allons greffer nos résultats à ceux des recherches antérieures pertinentes à notre objet d'étude afin de dresser le portrait de l'état des connaissances sur l'enseignement-apprentissage de l'improvisation musicale, bonifié par les apports de notre recherche.

\section{Compétences à développer chez l'apprenant}

Les compétences autodidactiques et synchrones que nous avons identifiées chez les participants de la présente recherche viennent étoffer les connaissances antérieures liées au volet psychologique de l'improvisation. L'importance accordée aux compétences synchrones par les musiciens improvisateurs que nous avons interviewés montre comment le fait que l'improvisation se déroule en temps réel implique un état d'esprit particulier lors de l'improvisation. En plus de la capacité de prendre des risques et de savoir accueillir la fausse note, deux compétences dont l'importance est soulignée dans la littérature antérieure (Dubé et Després 2012), notre recherche met en valeur d'autres compétences qui sont moins fréquemment abordées dans la littérature, telles que la capacité à faire preuve de "présence», d'ouverture et d'une capacité d'adaptation ainsi que la recherche d'une certaine concertation entre l'instinct et le savoir-faire et savoir. La primauté accordée aux compétences synchrones pourrait être expliquée par le fait que les musiciens de la présente étude, de par leur formation classique, 
définissent leur rapport à l'improvisation en l'opposant à l'interprétation, la pratique sur laquelle l'accent est généralement mis dans la pédagogie musicale classique traditionnelle. En effet, selon les dires des participants de la présente recherche, ces deux pratiques se traduisent par un rapport à l'inattendu et aux «erreurs» qui est complètement différent chez le musicien improvisateur que chez l'interprète. En effet, celui-ci recherche habituellement à offrir une prestation exempte d'erreurs, d'accrocs techniques ou d'évènements inattendus (Philippe, Thierry, Patricia, Lise, Sophie et Raúl), alors que celui-là doit plutôt apprendre à faire une utilisation musicale et créative de ces phénomènes.

Par ailleurs, les compétences musicales et théoriques que nous avons relevées sont reliées à l'apprentissage des règles musicales et de la technique instrumentale et à la capacité d'utiliser et d'adapter ses connaissances et ses habiletés musicales en temps réel. Bien que la plupart des compétences que nous avons identifiées dans cette catégorie aient été également mises de l'avant dans les recherches précédentes, la capacité de superposition thématique, l'intégration de l'approche d'analyse jazz aux connaissances antérieures et la direction d'orchestre ne sont pas apparues comme étant des compétences saillantes dans les recherches précédentes. En somme, nos résultats laissent entrevoir qu'une grande majorité des compétences que nous avons relevées seraient transversales aux différents genres musicaux où l'improvisation est pratiquée. Néanmoins, certaines compétences pourraient être spécifiques, ou tout au moins prépondérantes, à certains genres en raison du parcours d'apprentissage prédominant et des caractéristiques du langage musical improvisé propres à ce genre.

\section{Approches d'enseignement-apprentissage}

Alors qu'écouter les improvisateurs professionnels est un processus d'enculturation, les autres approches d'enseignement-apprentissage que nous avons recensées sont reliées à l'apprentissage des règles musicales et de la technique instrumentale, à la capacité d'utiliser et d'adapter ses connaissances et ses habiletés musicales en temps réel et à la mise en place de contraintes ou de règles d'improvisation. Alors que certaines de ces approches sont évoquées dans la littérature antérieure (notamment par Kenny et Gellrich 2002 et Thompson et Lehmann 2004), la liste que nous proposons ici est plus exhaustive et elle comporte certaines approches moins usuelles, telles que: travailler la laideur et la beauté du son, jouer en déplaçant des accents 9 , s'ancrer sur tonique-dominante, ériger la structure en temps réel, faire de l'improvisation générative et cultiver le sentiment d'urgence. Sans avoir la prétention de dresser un portrait exhaustif des pratiques pédagogiques reliées à l'improvisation musicale classique, nos résultats illustrent la vaste étendue des dimensions qui peuvent être travaillées pour et par l'improvisation. En effet, l'établissement des hauteurs de notes et des valeurs rythmiques en temps réel n'est que la conséquence audible d'une série complexe de processus cognitifs variés, agissant à différents niveaux.

9 Cette pratique propre au jazz, est très peu présente dans le contexte de la musique classique. 


\section{Structurer les éléments recensés : une proposition pédagogique}

Un des premiers grands constats sous-jacents à l'analyse de nos données est la grande transversalité qui existe entre nos résultats et ceux d'autres recherches qui ont été menées dans d'autres genres musicaux, tels que le jazz et l'improvisation libre. Cette transversalité laisse entrevoir que la pédagogie de l'improvisation musicale classique pourrait tirer profit des pratiques en vigueur dans d'autres genres musicaux, où l'improvisation occupe une part plus importante du curriculum. C’est pourquoi nous avons décidé de greffer nos résultats à ceux des recherches antérieures réalisées dans d'autres genres d'improvisation musicale. Ainsi, ce cadre pédagogique intègre les principaux résultats de notre recherche à notre synthèse de la littérature antérieure. 
Tableau 4. Cadre pédagogique pour l'enseignement-apprentissage de l'improvisation musicale classique

Connaissances identifiées dans la littérature

Connaissances issues des données de la recherche

\begin{tabular}{|c|c|c|}
\hline $\begin{array}{l}\text { Composantes de l'appren- } \\
\text { tissage de l'improvisation }\end{array}$ & $\begin{array}{l}\text { Approches } \\
\text { d'enseignement-apprentissage }\end{array}$ & Compétences à développer \\
\hline 1) Intérêt initiali & \multirow{2}{*}{$\begin{array}{l}\text { Écouter les improvisateurs } \\
\text { professionnels }\end{array}$} & \\
\hline \multirow[t]{2}{*}{ 2) Processus d'enculturationii } & & $\begin{array}{l}\text { - Audition intérieure } \\
\text { - Repiquage }\end{array}$ \\
\hline & \multirow[t]{3}{*}{ - Construire sa banque d'idées } & $\begin{array}{l}\text { - Connaissance de l'harmonie, } \\
\text { des gammes et des arpèges }\end{array}$ \\
\hline \multirow[t]{3}{*}{$\begin{array}{l}\text { 3) Apprentissage des règles } \\
\text { musicales et de la technique } \\
\text { instrumentale }\end{array}$} & & $\begin{array}{l}\text { - Reconnaissance de ses con- } \\
\text { naissances antérieures } \\
\text { - Transposition }\end{array}$ \\
\hline & & $\begin{array}{l}\text { Intégration de l'approche } \\
\text { d'analyse jazz aux con- } \\
\text { naissances antérieures }\end{array}$ \\
\hline & $\begin{array}{l}\text { - Travailler la laideur et la } \\
\text { beauté du son }\end{array}$ & \\
\hline \multirow{3}{*}{$\begin{array}{l}\text { 4) Capacité d'utiliser et d'adapt- } \\
\text { er ses connaissances et ses } \\
\text { habiletés musicales en temps } \\
\text { réeliv }\end{array}$} & - Direction d'orchestre & \\
\hline & $\begin{array}{l}\text { - Faire appel aux techniques } \\
\text { contrapuntiques }\end{array}$ & - Superposition thématique \\
\hline & \multirow{2}{*}{$\begin{array}{l}\text { - Faire de l'improvisation ryth- } \\
\text { mique pure } \\
\text { - Jouer en déplaçant des } \\
\text { - } \text { Faire de l'improvisation } \\
\text { mélodique } \\
\text { - Utiliser sa boîte d'idées } \\
\text { - Esquisser l'improvisation } \\
\text { - Poser des contraintes } \\
\text { - Focaliser sur les textures } \\
\text { - sonores } \\
\text { - Utiliser la gamme } \\
\text { chromatique } \\
\text { - S'ancrer sur } \\
\text { tonique-dominante } \\
\text { - Improviser sur une structure } \\
\text { - harmonique } \\
\text { - Faire de l'improvisation } \\
\text { formelle } \\
\text { - Ériger la structure en temps } \\
\text { réel } \\
\text { - Improviser dans le style de ... } \\
\text { Ornementer }\end{array}$} & \\
\hline $\begin{array}{l}\text { 5) Mise en place de contraintes } \\
\text { ou de règles d'improvisation }{ }^{\vee}\end{array}$ & & \\
\hline
\end{tabular}


Tableau 4 (suite)

\begin{tabular}{|c|c|c|}
\hline $\begin{array}{l}\text { Somposantes de l'apprentissage } \\
\text { de l'improvisation }\end{array}$ & $\begin{array}{c}\text { Approches } \\
\text { d'enseignement-apprentissage }\end{array}$ & Compétences à développer \\
\hline 6) Volet psychologiquevi & $\begin{array}{l}\text { - Faire de l'improvisation } \\
\text { "sans style» prédéfini } \\
\text { - Faire de l'improvisation } \\
\text { générative }\end{array}$ & \multirow{3}{*}{$\begin{array}{l}\text { - "Présence» } \\
\text { - Prise de risques } \\
\text { - Capacité d'adaptation } \\
\text { - Ouverture } \\
\text { - Concertation entre l'instinct et } \\
\text { - L'accueir-faire } \\
\text { - Motivation intrinsèque } \\
\text { - Habiletés métacognitives }\end{array}$} \\
\hline 7) Dimension socialevii & $\begin{array}{l}\text { - Jouer avec des musiciens } \\
\text { plus expérimentés }\end{array}$ & \\
\hline $\begin{array}{l}\text { 8) Apprentissages con- } \\
\text { crétisés dans un contexte } \\
\text { de concert devant public ou } \\
\text { d'enseignementviii }\end{array}$ & $\begin{array}{l}\text { - Cultiver le sentiment } \\
\text { d'urgence } \\
\text { - Faire de l'improvisation } \\
\text { interdisciplinaire }\end{array}$ & \\
\hline $\begin{array}{l}\text { 9) Développement d'un style } \\
\text { personne/ix }\end{array}$ & & Customisation \\
\hline \multicolumn{3}{|c|}{$\begin{array}{l}\text { i (Fraser 1983; Johansson 2008) } \\
\text { ii (Berliner 1994; Fraser 1983; Johansson 2008; Kenny et Gellrich 2002; Kingscott et } \mathrm{D} \\
\text { Lehmann 2004; Ward-Steinman 2014) } \\
\text { iii (Berkowitz 2009; Berliner 1994; Fraser 1983; Johansson 2008; Kenny et Gellrich } 200 \\
\quad \text { 2010) } \\
\text { iv (Berkowitz 2009; Berliner 1994; Kenny et Gellrich 2002) } \\
\text { v (Hickey 2015; Johnston 2013; Kenny et Gellrich 2002; Thompson et Lehmann 2004) } \\
\text { vi (Berliner 1994; Johansson 2008; Thompson et Lehmann 2004) } \\
\text { vii (Berliner 1994) } \\
\text { vii (Berkowitz 2009; Berliner 1994; Johansson 2008; Kenny et Gellrich 2002) } \\
\text { viii (Berkowitz 2009; Berliner 1994; Johansson 2008; Kenny et Gellrich 2002). } \\
\text { ix (Berliner 1994; Fraser 1983; Kratus 1995; Ward-Steinman 2014). }\end{array}$} \\
\hline
\end{tabular}

Ce cadre pédagogique repose sur une synthèse des connaissances scientifiques actuelles et des résultats issus de notre recherche et offre à celui qui s'intéresse à l'improvisation - qu'il soit apprenant ou enseignant, novice, débutant ou intermédiaire - des balises pour guider sa pratique instrumentale ou pédagogique. En effet, ce cadre pédagogique associe les compétences à développer chez l'apprenant ainsi que les approches d'enseignement-apprentissage qui ont été identifiées dans le cadre de la présente recherche aux diverses composantes de l'apprentissage de l'improvisation qui ont été identifiées dans les recherches antérieures pertinentes à notre objet d'étude.

Les compétences à développer chez l'apprenant improvisateur et les approches d'enseignement-apprentissage de l'improvisation que nous avons recensées représentent diverses pistes de réponses à notre question de recherche: "Quelles caractéristiques du parcours d'apprentissage ou de la pratique pédagogique des experts du domaine pourraient contribuer à bonifier l'enseignement-apprentissage de l'improvisation musicale classique?» Alors qu'il s'agit d'une des premières recherches empiriques dont le sujet central est l'approche d'enseignement des pédagogues experts et le processus d'apprentissage des instrumentistes experts en improvisation musicale classique qui soit fondée sur des données empiriques, l'efficacité de ce cadre pédagogique, ainsi que ses effets sur les autres dimensions du développement de l'apprenant musicien, devront être validés ultérieurement par des recherches empiriques expérimentales menées dans divers contextes d'enseignement-apprentissage de la musique classique. Par ailleurs, les trois manifestations de l'improvisation «improviser pour apprendre la musique», "apprendre à improviser musicalement » et «improviser musicalement pour apprendre» conceptualisées par Campbell (2009) 
illustrent bien comment l'improvisation peut s'inscrire en aval ou en amont de l'apprentissage musical et qu'elle peut aussi bien représenter un moyen qu'une fin pour l'apprenant ou le pédagogue. Ainsi, il serait intéressant de mesurer les effets du cadre pédagogique proposé sur les aptitudes instrumentales, personnelles (auto-efficacité, capacité de prise de risque, créativité, etc.) et sociales de l'apprenant. Finalement, des recherches ultérieures devraient être menées afin de documenter les pratiques pédagogiques réelles des enseignants experts en improvisation musicale classique dans leur contexte naturel. Cette piste de recherche permettrait notamment de faire l'analyse des dispositifs d'apprentissage, de l'aménagement des environnements, des contenus abordés, de l'ordre des activités proposées et du rôle joué par l'enseignant.

\section{RÉFÉRENCES}

Azzara, Christopher. D. 1992. "The Effect of Audiation-Based Improvisation Techniques on the Music Achievement of Elementary Instrumental Music Students ». Thèse de doctorat, University of Rochester : Rochester, NY.

Berkowitz, Aaron. 2009. "Cognition in Improvisation: The Art and Science of Spontaneous Musical Performance». Thèse de doctorat, Harvard University: Cambridge, MA.

Berliner, Paul F. 1994. Thinking in jazz: The Infinite Art of Improvisation. Chicago: University of Chicago Press.

Campbell, Patricia Shehan. 1991. «Unveiling the Mysteries of Musical Spontaneity». Music Educators Journal $78, \mathrm{n}^{\circ}$ 4: 21-4.

- 2009. "Learning to improvise music, improvising to learn music».Musical improvisation: Art, education, and society, sous la dir. de Gabriel Solis et Bruno Nettl, 119-42. Urbana, IL: University of Illinois Press.

Chi, Michelene. T. H. 2006. "Two approaches to the study of experts' characteristics». The Cambridge Handbook of Expertise and Expert Performance, sous la dir. de K. Anders Ericsson, Neil Charness, Paul J. Feltovich, et Robert R. Hoffman, 121-30. New York, NY: Cambridge University Press.

Després, Jean-Philippe et Francis Dubé. 2015. «Revue de littérature des écrits scientifiques portant sur l'improvisation musicale: identification des concepts clés et des recommandations pédagogiques liés à ce domaine». Cahiers de la Société Québécoise de Recherche en Musique 15, $\mathrm{n}^{\circ}$ 1: 55-66.

Dos Santos, R. A. T. et Luciana Del Ben. 2004. "Contextualized improvisation in solfège class ». International Journal of Music Education 22, $\mathrm{n}^{\circ} 3: 271-82$.

Dubé, Francis et Jean-Philippe Després. 2012. «Proposition d'un cadre conceptuel pour aider le professeur d'instrument à intégrer l'improvisation musicale à son acte pédagogique». Intersections, revue Canadienne de musique $32, \mathrm{n}^{\mathrm{o}} 1-2$ : 143-165.

Dubé, Francis, Isabelle Héroux et Noémie L. Robidas. 2015. «The factors that influence independent music teachers to use improvisation and composition activities, and music technologies with young beginners", The Reflective Conservatoire, 4th International Conference - creativity and Changing Culture, Londres. 
Dunn, Rita S. et Kenneth J. Dunn. 1979. «Learning Styles/Teaching Styles: Should they ... can they ... be Matched ». Educational Leadership $36, \mathrm{n}^{\circ} 4$ : $238-44$.

Ericsson, K. A. et N. Charness. 1994. "Expert performance: Its structure and acquisition. ", American Psychologist 49, $\mathrm{n}^{\mathrm{0}} 8$ : 725-47.

Fraser, Wilmot Alfred. 1983. "Jazzology: A study of the tradition in which jazz musicians learn to improvise». Thèse de doctorat, University of Pennsylvania: Philadelphia, PA.

Hickey, Maud. 2015. "Learning From the Experts A Study of Free-Improvisation Pedagogues in University Settings ». Journal of Research in Music Education 62, $\mathrm{n}^{\mathrm{O}} 4: 425-45$.

The Oxford Dictionary of Music. «Improvisation». http://www.oxfordmusiconline.com/subscriber/article/opr/t237/e5140

Johansson, Karin. 2008. "Organ improvisation-activity, action and rhetorical practice». Thèse de doctorat, Malmö Academy of Music, Lund University: Malmö.

Johnston, Peter. 2013. "Teaching improvisation and the pedagogical history of the Jimmy Giuffre $3 »$. International Journal of Music Education 31, $\mathrm{n}^{\circ} 4$ : 383-93.

Kenny, Barry. J. et Martin Gellrich. 2002. "Improvisation». The Science and Psychology of Music Performance, sous la dir. Richard Parncutt et Gary McPherson, 117-34. New York: Oxford University Press.

Kingscott, John et Colin Durrant. 2010. "Keyboard improvisation: a phenomenological study». International Journal of Music Education 28, $\mathrm{n}^{0} 2$ : 127-44.

Koutsoupidou, Theano et David J. Hargreaves. 2009. "An experimental study of the effects of improvisation on the development of children's creative thinking in music». Psychology of Music $37, \mathrm{n}^{\mathrm{0}} 3: 251-78$.

Kratus, John. 1991. "Growing with improvisation». Music Educators Journal $78, \mathrm{n}^{\circ} 4: 35-40$.

-1995. "A developmental approach to teaching musical improvisation». International Journal of Music Education 19, $\mathrm{n}^{\circ}$ 1: 35-50.

Lange, Barbara Rose. 2011. "Teaching the Ethics of Free Improvisation». Critical Studies in Improvisation/Études critiques en improvisation $7, \mathrm{n}^{0} 2$. http://www.criticalimprov.com/article/view/964

Lemaire, Patrick et Robert S. Siegler. 1995. «Four aspects of strategic change: Contributions to children's learning of multiplication. ». Journal of Experimental Psychology 124, $\mathrm{n}^{\circ}$ 1: 83-97.

Manderson, Desmond. 2010. «Fission and Fusion: From improvisation to formalism in law and music». Critical Studies in Improvisation/Études critiques en improvisation $6, \mathrm{n}^{0}$ 1. http://www.criticalimprov.com/article/ viewArticle/1167/1726

McPherson, Gary E. 1993. "Evaluating Improvisational Ability of High School Instrumentalists». Bulletin of the Council of Research in Music Education 119: 11-20. 
McPherson, Gary. E., Michael Bailey et Kenneth E. Sinclair. 1997. «Path analysis of a theoretical model to describe the relationship among five types of musical performance». Journal of Research in Music Education 45, $\mathrm{n}^{\mathrm{0}} 1: 103-29$.

Montano, David R. 1983. The effect of improvisation in given rhythms on rhythmic accuracy in sight reading achievement by college elementary group piano students. Thèse de doctorat, Conservatory of Music, University of Missouri: Kansas City.

Moore, Robin. 1992. "The Decline of Improvisation in Western Art Music: An Interpretation of Change». International Review of the Aesthetics and Sociology of Music 23, $\mathrm{n}^{\mathrm{o}}$ 1: 61-84.

Moreira, Lídia et Sara Carvalho. 2010. «Exploration and improvisation: the use of creative strategies in instrumental teaching». International Journal for Cross-Disciplinary Subjects in Education 1: 248-54.

Nehls, Kimberly, Brandy D. Smith et Holly A. Schneider. 2015. «Video-Conferencing Interviews in Qualitative Research». Enhancing Qualitative and Mixed Methods Research with Technology, sous la dir. de Shalin Hai-Jew, 140-57. IGI Global: Hershey.

Norgaard, Martin. 2011. "Descriptions of improvisational thinking by artist-level jazz musicians». Journal of Research in Music Education 59, $\mathrm{n}^{\mathrm{o}} 2: 109-27$.

Paillé, Pierre et Alex Mucchielli. 2012. L'analyse qualitative en sciences humaines et sociales. Paris: Armand Colin.

Pignato, Joseph. 2013. "Angelica gets the spirit out: Improvisation, epiphany and transformation». Research Studies in Music Education 35, $\mathrm{n}^{\circ}$ 1: 21-38.

Plucker, Jonathan A., Mark A. Runco et C. Boyd Hegarty. 2011. "Enhancement of Creativity». Dans Encyclopedia of Creativity (Second Edition), sous la dir. de Mark A. Runco et Steven R. Pritzker, 456-46o. Academic Press: San Diego.

Scott, Julie K. 2007. «Me? Teach Improvisation to Children?». General Music Today $20, \mathrm{n}^{\mathrm{O}} 2: 6-13$.

Sloboda, John. A. 1996. "The acquisition of musical performance expertise: Deconstructing the "talent" account of individual differences in musical expressivity». The Road To Excellence: The Acquisition of Expert Performance in the Arts and Sciences, Sports, and Games, sous la dir. de K. Anders Ericsson, 107-26. Psychology Press: New York.

—. 1993. "Musical Ability». Ciba Foundation Symposium 178-The Origins and Development of High Ability sous la dir. de Gregory R. Bock et Kate Ackrill, 106-18. John Wiley \& Sons, Ltd.: Chichester.

«Soundpainting». http://www.soundpainting.com/soundpainting (consulté le 14 janvier 2016).

Thompson, Sam et Andreas C. Lehmann. 2004. "Strategies for Sight-Reading and Improvising Music». Musical excellence: Strategies and Techniques to Enhance Performance, sous la dir. de Aaron Williamon, 143-59. Oxford: Oxford University Press . 
Ward-Steinman, Patrice Madura. 2014. "The vocal improviser-educator: An analysis of selected American and Australian educators' influences and pedagogical views». International Journal of Music Educcation $32, \mathrm{n}^{\circ} 3$ : 346-59.

Whitman, Georann G. 2001. "The effects of vocal improvisation on attitudes, aural identification skills, knowledge of music theory, and pitch accuracy in sight-reading of high school choral singers». Mémoire de maîtrise, Conservatory of Music. University of Missouri: Kansas City.

Wilson, Dorothy Stevens. 1970. "A Study of the Child Voice from Six to Twelve». Thèse de doctorat, University of Oregon: Eugene.

\section{RÉSUMÉ}

Cette recherche porte sur l'enseignement-apprentissage de l'improvisation musicale classique. Afin d'offrir une perspective en profondeur de cet objet d'étude, des entrevues semi-dirigées ont été menées auprès de $\mathrm{N}=15$ participants, au sujet de leur processus d'apprentissage, de production et d'enseignement de l'improvisation. Différentes composantes de l'enseignement-apprentissage de l'improvisation musicale classique ont été identifiées: 18 compétences à développer chez l'apprenant et 23 approches d'enseignement-apprentissage. Ces connaissances offrent à celui qui s'intéresse à l'improvisation - qu'il soit enseignant ou apprenant, de niveau novice, débutant ou intermédiaire - des balises pour guider son apprentissage instrumental ou sa pratique pédagogique.

\section{ABSTRACT}

This research focuses on the teaching and learning of classical music improvisation. In order to provide an in-depth perspective on the subject, semi-directed interviews were led with $\mathrm{N}=15$ participants, exploring their learning, teaching and production processes of improvisation. A number of components have been identified in such processes which corresponds to 18 skills the learner has to develop, and 23 teaching and learning approaches. These observations and analyses constitutes to those interested in music improvisation-whether as learner or teacher of all levels-concrete guidelines to help in their learning or in their teaching methods.

\section{BIOGRAPHIE}

Jean-Philippe Després (Ph. D.) mène actuellement un projet de recherche postdoctoral (Mitacs Élévation) visant l'élaboration d'outils pédagogiques novateurs en enseignement musical et l'intégration de ces outils dans le milieu pratique. Son projet doctoral (FRQ-SC, CRSH et OICRM) - portant sur le parcours d'apprentissage, les stratégies de création et les approches d'enseignement des improvisateurs experts en musique classique - a donné le jour à diverses publications, notamment dans les revues scientifiques Journal of Research in Music Education et Thinking Skills and Creativity. JeanPhilippe a étudié la guitare classique auprès de Michel Caron (Canada), Sebastián Guigui (Mexique), Alejandro Mora (Mexique), Rémi Boucher (Canada), Massimo 
Gatta (Italie) et Isabelle Héroux (Canada), entre autres. Par ailleurs, il joue du oud et de la guitare électrique dans des ensembles expérimentaux et de musique du monde depuis plus de 20 ans. Ses principaux centres d'intérêt sont l'enseignement-apprentissage de la guitare, la créativité, l'optimisation de l'apprentissage, l'improvisation, les TIC liées à la musique et l'apprentissage par le jeu. 